\title{
Center-symmetric effective theory for two-color QCD at high and moderate temperature
}

\section{Tomáš Brauner}

Faculty of Physics, University of Bielefeld, Germany

Department of Theoretical Physics, Nuclear Physics Institute ASCR, Řež, Czech Republic

E-mail: tbrauner@physik.uni-bielefeld.de

\begin{abstract}
We revisit the center-symmetric dimensionally reduced effective theory for two-color Yang-Mills theory at high temperature. This effective theory includes an order parameter for deconfinement and thus allows to broaden the range of validity of the conventional three-dimensional effective theory (EQCD) towards the confining phase transition. We extend the previous results by including the effects of massive quarks with nonzero baryon chemical potential. The parameter space of the theory is constrained by leading-order matching to the Polyakov loop effective potential of two-color QCD. Once all the parameters are fixed, the effective theory can provide modelindependent predictions for the physics above the deconfinement transition, thus bridging the gap between large-scale numerical simulations and semi-analytical calculations within phenomenological models.
\end{abstract}

Xth Quark Confinement and the Hadron Spectrum

8-12 October 2012

TUM Campus Garching, Munich, Germany 
Understanding the phase diagram of quantum chromodynamics (QCD) is a major challenge for current particle physics. Partial information can be obtained using ab initio numerical simulations on the lattice, which are complemented by experimental data from relativistic heavy-ion collisions. However, both of these approaches are constrained to the region of low net baryon density, in the case of lattice simulations due to the infamous sign problem, and in the case of heavy-ion collisions as a result of the particular experimental set-up. The interpretation of the latter is further complicated by the fact that what one observes is a strongly non-equilibrium system. In addition, astrophysical observations can provide some input on the region of the phase diagram corresponding to low temperature and moderate density. (What is low and what is high is determined by the characteristic energy scale of the strong interaction, $\Lambda_{\mathrm{QCD}} \simeq 300 \mathrm{MeV}$.)

Analytic first-principle approaches are invariably based on series expansion in a given small parameter, and thus provide reliable computation schemes only in specific limits. In the case of the chiral perturbation theory, the expansion parameter is momentum or energy, and its applicability is therefore limited to the low-temperature and low-density corner of the phase diagram. On the other hand, there are several thermal field theory methods, such as perturbation theory and its various modifications, that are based on expansion in the QCD coupling $g$, and hence are limited to very high temperatures. Model approaches can in principle cover the whole phase diagram. Their main disadvantage lies in the fact that they by construction cannot reproduce all features of QCD.

From the above, it is clear that existing first-principle analytic calculations and lattice simulations are complementary, in both the range of applicability and the computation effort. In this contribution, we report on an attempt to bridge the gap between them. We construct an approach that is semi-analytic and, by exploiting the symmetry of QCD, has a range of validity that extends down to the critical temperature for (de)confinement. It thus has the potential to provide a link between the perturbative methods and lattice simulations.

\section{Introduction}

Our approach is based on three main ingredients that will be briefly introduced below: dimensional reduction, center symmetry, and two-color QCD. In the imaginary time formalism, thermal gluons acquire an effective mass equal to $2 \pi n T$ where $n$ is an integer and $T$ the temperature. At very high temperature, the $n=0$ mode is the only one that does not have a parametrically large mass. The original four-dimensional field theory then reduces to a three-dimensional theory for this soft mode, a fact usually dubbed dimensional reduction.

In a pure gauge $\mathrm{SU}(N)$ theory, the (de)confinement is a genuine phase transition. This transition is associated with the change of symmetry of the system. In the high-temperature, deconfined phase the center symmetry is spontaneously broken, while in the low-temperature, confined phase it is restored. The corresponding order parameter is provided by the expectation value of the Polyakov loop. In presence of dynamical quarks, the center symmetry is violated, yet it is known to still play an important role in the dynamics of the transition.

For technical reasons to be explained later, we restrict our attention to Yang-Mills theory with two colors and quarks in the fundamental representation, in short two-color $Q C D$. The deconfinement phase transition in the $\mathrm{SU}(2)$ Yang-Mills theory is known to be second order, and the $\mathrm{Z}_{2}$ center symmetry thus plays a key role, leading to critical behavior in the correct universality class. 
The generalization of our construction to the real, three-color QCD is in principle straightforward, the only obstacle being the large number of free parameters in the effective theory that need to be determined by perturbative matching.

\subsection{Dimensional reduction}

In the imaginary time formalism, quantum field theory at nonzero temperature can be thought of as a theory with the temporal dimension compactified to a circle with circumference $1 / T$. The spectrum then consists of a Kaluza-Klein tower of modes. At very high temperature, all nonzero Matsubara modes can be integrated out, resulting in a purely three-dimensional effective theory for the zero modes of the bosonic fields. (Fermions do not have any zero modes and thus are completely integrated out.)

In the context of QCD, the resulting effective theory is called Electrostatic QCD (EQCD) and has been investigated in detail in refs. [1,2]. Its degrees of freedom are the spatial gluon $A_{i}^{a}$ and the temporal gluon $A_{0}^{a}$ that transforms under three-dimensional gauge transformations as an adjoint scalar. Due to thermal fluctuations, this mode acquires a nonzero screening (Debye) mass of the order $g T$, where $g$ is the four-dimensional gauge coupling. The resulting EQCD Lagrangian reads, to the lowest order,

$$
\mathscr{L}_{\mathrm{EQCD}}=\frac{1}{4}\left(F_{i j}^{a}\right)^{2}+\frac{1}{2}\left(D_{i} A_{0}^{a}\right)^{2}+\frac{1}{2} m_{E}^{2}\left(A_{0}^{a}\right)^{2}+\frac{1}{8} \lambda_{E}\left(A_{0}^{a} A_{0}^{a}\right)^{2},
$$

where $D_{i} \equiv \partial_{i}-\mathrm{i}\left[A_{i}, \cdot\right]$ is the covariant derivative. The parameters of the effective Lagrangian can be determined by a direct perturbative matching. For $N_{\mathrm{f}}$ flavors of massless quarks in two-color QCD, they take the form

$$
m_{E}^{2}=\frac{2 g^{2} T^{2}}{3}\left(1+\frac{N_{\mathrm{f}}}{4}\right), \quad \lambda_{E}=\frac{2 g^{4} T}{3 \pi^{2}}\left(1-\frac{N_{\mathrm{f}}}{8}\right) .
$$

Analytic expressions for quarks with arbitrary masses are available in ref. [3].

\subsection{Center symmetry}

Center symmetry is a global $Z_{N}$ symmetry of the thermal $\mathrm{SU}(N)$ Yang-Mills theory associated with the nontrivial topology of the compactified spacetime. The corresponding order parameter is given by the Polyakov loop,

$$
\Omega(\boldsymbol{x}) \equiv \mathscr{P} \exp \left[\mathrm{i} g \int_{0}^{\beta} \mathrm{d} \tau A_{0}(\tau, \boldsymbol{x})\right] .
$$

At high temperatures when the center symmetry is spontaneously broken, the expectation value of the Polyakov loop is localized around one of $N$ physically equivalent equilibrium states, related to one another by $\mathrm{Z}_{N}$ transformations. The EQCD Lagrangian (1.1) is based on an expansion in powers of $A_{0}^{a}$ and hence violates the center symmetry. This is appropriate at very high temperatures when the tunneling transitions between different $Z_{N}$ states are strongly suppressed. However, as the temperature decreases towards the confinement transition, fluctuations of the Polyakov loop become important and picking just one of the degenerate equilibrium states is no longer a good 
approximation. This is one of the reasons why EQCD fails at temperatures near the confinement transition.

In order to extend the range of applicability of the analytic weak-coupling methods to lower temperatures, a formalism was proposed in refs. $[4,5,6]$ which preserves center symmetry explicitly. This center-symmetric dimensionally reduced effective theory (ZQCD) should be understood as an improvement of EQCD. As the low-energy degree of freedom containing the screened soft modes, the temporal gluon $A_{0}^{a}$ is replaced with the Polyakov loop, smeared over a length scale of order $1 / T$. In refs. $[4,5]$, the formalism was worked out for the pure gauge SU(3) Yang-Mills theory, while in ref. [6] it was applied to the case of two colors. The main aim of this contribution is to extend the effective theory by incorporating the effects of dynamical quarks with arbitrary masses and chemical potentials.

\subsection{Two-color QCD}

Two-color QCD is not only a simpler cousin of the real, three-color QCD; it is attractive for several reasons. First, it does not suffer from the sign problem and thus is amenable to lattice simulations at nonzero chemical potential [7]. Second, at high baryon density, its thermodynamics is, like in three-color QCD, driven by the Fermi sea of quarks, while the low-energy physics is governed by Cooper pairing of quarks near the Fermi surface. Unlike in the three-color case, however, the Cooper pair is a color singlet and thus provides a gauge-invariant order parameter: cold and dense two-color QCD is a baryonic superfluid.

The last observation has far-reaching consequences also for the low-density thermodynamics and even for the structure of the vacuum. The fact that two quarks can form a color singlet state means that baryons in two-color QCD are bosons. The onset of nonzero baryon density (at zero temperature) is thus marked by Bose-Einstein condensation of diquark baryons rather than by the appearance of a Fermi sea of nucleons. This among others implies dramatic simplification of models for "nuclear physics"; one avoids the complications of three-body physics in nuclear matter.

On the level of the Lagrangian, the above properties follow from the Pauli-Gürsey symmetry between quarks and antiquarks [8]. This results in the embedding of the usual chiral group $\mathrm{SU}\left(N_{\mathrm{f}}\right)_{\mathrm{L}} \times \mathrm{SU}\left(N_{\mathrm{f}}\right)_{\mathrm{R}} \times \mathrm{U}(1)_{\mathrm{B}}$ in a simple flavor symmetry group $\mathrm{SU}\left(2 N_{\mathrm{f}}\right)$. This naturally includes symmetry transformations that change baryon number so that the multiplets of the symmetry contain the baryonic diquarks as well as mesons. In the past decade, much effort has been devoted to the understanding of the phase structure of two-color QCD using lattice simulations [7], effective field theory [9], as well as model calculations [10]. We complement the existing results by providing a framework for investigating the thermodynamics of two-color QCD at high and moderate temperatures and low density.

\section{Center-symmetric effective theory}

As emphasized above, we aim at constructing an effective theory for two-color QCD which preserves the $Z_{2}$ center symmetry and at the same time reduces to EQCD at high temperature where the effects of center symmetry are negligible. Moreover, we demand for purely technical reasons that the theory be superrenormalizable. This leads to technical simplifications when simulating the nonperturbative magnetic part of the theory on the lattice $[5,6]$. The relevant parameters of the 
theory will be fixed by perturbative matching. We review the construction of the theory according to ref. [6], taking into account the effects of dynamical quarks.

The degrees of freedom of ZQCD are the spatial gluon $A_{i}^{a}(\boldsymbol{x})$ and the coarse-grained Polyakov loop field $\mathscr{Z}(\boldsymbol{x})$. They undergo the usual gauge transformations

$$
\mathscr{Z}(\boldsymbol{x}) \rightarrow U(\boldsymbol{x}) \mathscr{Z}(\boldsymbol{x}) U(\boldsymbol{x})^{\dagger}, \quad \boldsymbol{A}(\boldsymbol{x}) \rightarrow U(\boldsymbol{x})[\boldsymbol{A}(\boldsymbol{x})+\mathrm{i} \boldsymbol{\nabla}] U(\boldsymbol{x})^{\dagger},
$$

where $U(\boldsymbol{x}) \in \mathrm{SU}(2)$. In addition, the $\mathscr{Z}$ field is subject to the center symmetry transformation as $\mathscr{Z}(\boldsymbol{x}) \rightarrow \pm \mathscr{Z}(\boldsymbol{x})$. The technical simplification particular to the $\mathrm{SU}(2)$ group is that a linear combination of unitary matrices is unitary up to an overall factor. Thus, the coarse-grained Polyakov loop $\mathscr{Z}(\boldsymbol{x})$ can be parameterized as

$$
\mathscr{Z}(\boldsymbol{x})=\frac{1}{2}\left[\Sigma(\boldsymbol{x})+\mathrm{i} \sigma_{a} \Pi_{a}(\boldsymbol{x})\right],
$$

where $\sigma_{a}$ are Pauli matrices while $\Sigma$ and $\Pi_{a}$ are real scalar fields. The SU(2) adjoint field $\Pi_{a}$ corresponds to the light adjoint field $A_{0}^{a}$ in EQCD, while $\Sigma$ encodes fluctuations in the magnitude of the Polyakov loop and is expected to get a mass of the order of the temperature.

The most general Lagrangian density with operators up to fourth order in the fields, consistent with the three-dimensional gauge invariance and rotational invariance, takes the form

$$
\mathscr{L}=\frac{1}{g_{3}^{2}}\left[\frac{1}{2} \operatorname{Tr} F_{i j}^{2}+\operatorname{Tr}\left(D_{i} \mathscr{Z}^{\dagger} D_{i} \mathscr{Z}\right)+V(\mathscr{Z})\right],
$$

where $g_{3}$ is the gauge coupling in the dimensionally reduced theory and the potential $V(\mathscr{Z})$ reads

$$
V(\mathscr{Z})=b_{1} \Sigma^{2}+b_{2} \Pi_{a}^{2}+c_{1} \Sigma^{4}+c_{2}\left(\Pi_{a}^{2}\right)^{2}+c_{3} \Sigma^{2} \Pi_{a}^{2}+d_{1} \Sigma^{3}+d_{2} \Sigma \Pi_{a}^{2} .
$$

Note that the $d_{1,2}$ operators explicitly break the $Z_{2}$ center symmetry, and thus incorporate the effects of dynamical quarks. Of course, the presence of quarks also affects the values of the centerpreserving couplings in the Lagrangian.

As stressed above, we expect the masses of $\Sigma$ and $\Pi_{a}$ to be parametrically of different orders, $T$ and $g T$, respectively. To achieve this, we rewrite the potential $V(\mathscr{Z})$ as

$$
V(\mathscr{Z})=h_{1} \operatorname{Tr}\left(\mathscr{Z}^{\dagger} \mathscr{Z}\right)+h_{2}\left(\operatorname{Tr} \mathscr{Z}^{\dagger} \mathscr{Z}\right)^{2}+g_{3}^{2}\left[\frac{s_{1}}{2} \Pi_{a}^{2}+\frac{s_{2}}{4}\left(\Pi_{a}^{2}\right)^{2}+s_{3} \Sigma^{4}+\frac{s_{4}}{2} \Sigma^{3}+\frac{s_{5}}{2} \Sigma \Pi_{a}^{2}\right] .
$$

The "hard" part of the potential now has an extended $\mathrm{SU}(2)_{\mathrm{L}} \times \mathrm{SU}(2)_{\mathrm{R}}$ symmetry. This is spontaneously broken by the nonzero expectation value of $\mathscr{Z}$ in the high-temperature phase where center symmetry is broken. The $\Pi_{a}$ are interpreted as the corresponding pseudo-Nambu-Goldstone modes that only acquire nonzero mass from the "soft" part of the potential.

\subsection{Perturbative matching}

In order to fix the parameters of the effective theory, we have to compare its predictions with those of the full theory, two-color QCD. To that end, we keep only the physical degrees of freedom $\Pi_{a}$ and integrate out the auxiliary mode $\Sigma$. To the order that we are interested in, this can be done 
simply by using its equation of motion. The result is to be compared to the one-loop effective potential for the Polyakov loop in the full theory [11],

$$
\begin{aligned}
V_{\mathrm{eff}}\left(\Pi_{a}\right)= & \frac{4}{3} \pi^{2} T^{4}\left\langle\frac{g|\boldsymbol{\Pi}|}{2 \pi T}\right\rangle^{2}\left(1-\left\langle\frac{g|\boldsymbol{\Pi}|}{2 \pi T}\right\rangle\right)^{2}+ \\
& +\frac{4 T^{2}}{\pi^{2}} \sum_{j=1}^{N_{\mathrm{f}}} m_{j}^{2} \sum_{n=1}^{\infty} \frac{(-1)^{n}}{n^{2}} K_{2}\left(n \beta m_{j}\right) \cosh \left(n \beta \mu_{j}\right) \cos \frac{n g|\boldsymbol{\Pi}|}{2 T},
\end{aligned}
$$

where the angular brackets denote the integer part. The masses $m_{j}$ and chemical potentials $\mu_{j}$ of the individual quark flavors can in principle be arbitrary. However, the above infinite series only converges provided $\mu_{j}<m_{j}$ for all flavors.

Apart from the effective coupling $g_{3}$, which is to the leading order determined by its counterpart in the full theory as $g_{3}^{2}=g^{2} T$, the effective potential (2.5) contains altogether seven unknown parameters, namely $h_{1,2}$ and $s_{1-5}$. One combination of the hard parameters is determined by the size of the $\Sigma$ condensate in the effective theory, $v_{0}=2 T$, which to the leading order leads to the constraint $h_{1}+4 T^{2} h_{2}=0$. The other hard parameter cannot be found by perturbative matching and has to be fixed by nonperturbative lattice simulation [6]. Fortunately, it has, as expected, little influence on the physics at the soft scale $g T$.

Among the five soft parameters, two linear combinations of $s_{1-3}$ are obtained by matching to the EQCD Lagrangian (1.1). This is equivalent to comparing the coefficients of the Taylor expansion of the Weiss potential (2.6) around zero. This leads to the constraints

$$
m_{E}^{2}=g_{3}^{2}\left(s_{1}-4 s_{3} v_{0}^{2}-\frac{3}{2} s_{4} v_{0}+s_{5} v_{0}\right), \quad \lambda_{E}=2 g_{3}^{4}\left(s_{2}+4 s_{3}+\frac{3 s_{4}}{4 v_{0}}-\frac{s_{5}}{v_{0}}\right) .
$$

Once again, the remaining linear combination of $s_{1-3}$ cannot be fixed by matching of low-energy observables. This can be understood from the observation that integrating out the heavy mode $\Sigma$ effectively reduces the number of independent operators in the Lagrangian. This unknown parameter does not affect low-energy physics to the order that we work at.

Finally, the parameters $s_{4,5}$ encode the effects of explicit center symmetry breaking due to finite quark masses and thus require the knowledge of the global structure of the Weiss potential (2.6). In ref. [3], they were determined using the difference of selected observables in the absolute, stable minimum and the metastable minimum of the Weiss potential.

\subsection{Predictions of the theory}

Once all the necessary parameters are fixed, the effective theory can be used to make predictions. Some initial consistency test were performed in ref. [6] where the $Z_{2}$ phase transition in the effective theory was studied and its universality properties were checked.

In order to clearly discriminate ZQCD from its center-symmetry-violating predecessor, $\mathrm{EQCD}$, it is most illuminating to consider observables that are sensitive to the global structure of the Weiss potential. In the absence of dynamical quarks, the potential has two degenerate minima and the theory possesses a stable topological configuration, the domain wall. In full QCD, the domain wall solution was found, at one loop, in ref. [12]. Within the effective theory, the field configuration minimizing free energy clearly satisfies the constraint $\Sigma^{2}+\Pi_{a}^{2}=v_{0}^{2}$. The fields can then be parameterized by an angle parameter $\alpha$, ranging from zero to one, as $\Sigma=v_{0} \cos (\pi \alpha),|\boldsymbol{\Pi}|=v_{0} \sin (\pi \alpha)$. 
The correct evaluation of the resulting effective potential requires the calculation of one-loop corrections, leading to the expression

$$
\begin{aligned}
V_{\mathrm{eff}}(\alpha)= & \frac{v_{0}^{2}}{2}\left(s_{1}-4 s_{3} v_{0}^{2}\right) \sin ^{2}(\pi \alpha)+\frac{v_{0}^{4}}{4}\left(s_{2}+4 s_{3}\right) \sin ^{4}(\pi \alpha)-\frac{v_{0}^{3}}{3 \pi}|\sin (\pi \alpha)|^{3}+ \\
& +\frac{v_{0}^{3}}{2} s_{4}\left[\cos ^{3}(\pi \alpha)-1\right]+\frac{v_{0}^{3}}{2} s_{5} \cos (\pi \alpha) \sin ^{2}(\pi \alpha) .
\end{aligned}
$$

In the absence of $Z_{2}$ breaking effects, this potential depends on two linear combinations of the soft effective couplings $s_{1-3}$, which are fixed by the matching conditions (2.7). The profile of the domain wall is thus a sheer prediction of the effective theory. In particular, the domain wall tension takes the value (see ref. [3] for details of the calculation)

$$
\sigma \approx 4.899 \times \frac{T^{3}}{g} \approx 0.91 \sigma_{\mathrm{YM}}
$$

where $\sigma_{\mathrm{YM}}=\left(\frac{2}{3}\right)^{3 / 2} \frac{\pi^{2} T^{3}}{g}$ denotes the full theory result [12].

Once center symmetry is violated by the presence of dynamical quarks, the domain is no longer stable. However, there is still a static spherically-symmetric three-dimensional solution to the equations of motion, which corresponds to a bubble of the stable phase in the medium of the metastable one. In case of weak explicit breaking of center symmetry (that is, large quark masses), the bubble profile can be found analytically in the so-called thin-wall approximation. The actual critical radius of the bubble results from the competition of surface energy cost and bulk energy gain. In this approximation, it is determined solely by the surface (domain-wall) tension and energy density difference between the stable and the metastable phase. The radius and the action of the bubble are given by the following universal expressions, valid in both the full theory and in ZQCD,

$$
R_{c}=\frac{2}{\delta} \times \frac{\sigma}{T^{3} / g}, \quad S_{\text {bubble }}=\frac{16 \pi}{3 g^{3} \delta^{2}} \times\left(\frac{\sigma}{T^{3} / g}\right)^{3} .
$$

where $\sigma$ is the surface tension and $\delta$ the energy density difference expressed in units of $T$.

\section{Summary and outlook}

We have constructed an effective theory for two-color QCD with dynamical quarks, valid at moderate-to-high temperatures. Preserving by construction the center symmetry of QCD, this theory contains all the ingredients necessary for a qualitatively correct description of thermodynamics near the (de)confinement phase transition. Parameters that are relevant for physics at the soft scale $g T$ are fixed by matching to the Weiss potential of the full theory. Compared to ref. [6], we have included the effects of dynamical quarks with arbitrary masses and chemical potentials. We have thus prepared the stage for a systematic exploration of the phase diagram of two-color QCD at high temperature by effective field theory methods.

As a first application, we calculated the tension of the domain wall, stretching between the two minima of the Weiss potential. This is a pure prediction of the effective theory, and at the lowest order in the weak coupling, it differs from the result in the full theory by about $9 \%$. Next, 
the effective theory can be used to study thermodynamics of two-color QCD in the range of temperatures and densities where center symmetry is expected to be important. In particular, since the dependence of the effective couplings on quark chemical potentials is known, analytic continuation to imaginary chemical potential is trivial. Our effective theory should then be able to reproduce the Roberge-Weiss transition correctly.

\section{Acknowledgments}

The results presented here were obtained in collaboration with A. Kurkela, A. Vuorinen and T. Zhang. This work was supported by the Sofja Kovalevskaja program of the Alexander von Humboldt Foundation.

\section{References}

[1] K. Kajantie, M. Laine, K. Rummukainen, and M. E. Shaposhnikov, Generic rules for high temperature dimensional reduction and their application to the Standard Model, Nucl. Phys. B458 (1996) 90-136, [hep-ph/9508379].

[2] E. Braaten and A. Nieto, Free energy of QCD at high temperature, Phys. Rev. D53 (1996) 3421-3437, [hep-ph/9510408].

[3] T. Zhang, T. Brauner, A. Kurkela, and A. Vuorinen, Two-color QCD via dimensional reduction, JHEP 02 (2012) 139, [arXiv:1112.2983].

[4] A. Vuorinen and L. G. Yaffe, Z(3)-symmetric effective theory for SU(3) Yang-Mills theory at high temperature, Phys. Rev. D74 (2006) 025011, [hep-ph / 0604100 ].

[5] A. Kurkela, Framework for non-perturbative analysis of a Z(3)-symmetric effective theory of finite temperature QCD, Phys. Rev. D76 (2007) 094507, [arXiv: 0704 . 1416].

[6] P. de Forcrand, A. Kurkela, and A. Vuorinen, Center-symmetric effective theory for high-temperature SU(2) Yang-Mills theory, Phys. Rev. D77 (2008) 125014, [arXiv: 0801.1566 ].

[7] S. Cotter, P. Giudice, S. Hands, and J.-I. Skullerud, Towards the phase diagram of dense two-color matter, arXiv:1210.4496.

[8] A. Smilga and J. J. M. Verbaarschot, Spectral sum rules and finite volume partition function in gauge theories with real and pseudoreal fermions, Phys. Rev. D51 (1995) 829-837, [hep-th/ 9404031 ].

[9] K. Splittorff, D. Toublan, and J. J. M. Verbaarschot, Thermodynamics of chiral symmetry at low densities, Nucl. Phys. B639 (2002) 524-548, [hep-ph/ 0204076$].$

[10] C. Ratti and W. Weise, Thermodynamics of two-color QCD and the Nambu Jona-Lasinio model, Phys. Rev. D70 (2004) 054013, [hep-ph/ 0406159 ].

[11] N. Weiss, Effective potential for the order parameter of gauge theories at finite temperature, Phys. Rev. D24 (1981) 475-480.

[12] T. Bhattacharya, A. Gocksch, C. Korthals Altes, and R. D. Pisarski, Interface Tension in an $S U(N)$ Gauge Theory at High Temperature, Phys. Rev. Lett. 66 (1991) 998-1000. 